\title{
Forgetting Bits and Pieces
}

\section{An Exploration of the "Right to Be Forgotten" as Implementation of "Forgetting" in Online Memory Processes}

\author{
Paulan Korenhof \\ Privacy \& Identity lab \\ Tilburg Institute for Law, Technology, and Society (TILT) \\ Tilburg University, Tilburg, the Netherlands \\ p.e.i.korenhof@tilburguniversity.edu
}

\begin{abstract}
Technology has changed and still is changing our internal and external memory processes. The World Wide Web (Web) can function as an external transactive memory and can store and provide access to personal information for a very long time. The "right to be forgotten or erasure" (R2BFE), article 17 of the proposed General Data Protection Regulation, aims at helping individuals to control the availability of online accessible personal information. This paper takes the term "forgetting" in the article's title seriously and reviews the manner in which the R2BFE implements "forgetting" into the transactive memory on the Web. Exploring the concept of forgetting in this context shows that there is a far broader scale of options to implement digital forgetting than is offered today by the R2BFE. The analysis shows where the R2BFE is insufficient and risks affecting other interests at stake more than is necessary by the application of too narrow a notion of forgetting. This paper suggests that the R2BFE could be transformed into a more successful implementation of "forgetting" in the online transactive memory if it were to draw more heavily on the mechanisms of human forgetting.
\end{abstract}

Keywords: Data protection, forgetting, Internet, memory, right to be forgotten.

\section{Introduction}

"The Web Means the End Of Forgetting" [15]: The title of this 2010 article by Rosen expresses the growing concern with personal data available on the World Wide Web $(\mathrm{Web})^{1}$ and the potential consequences of permanent availability of these data for individuals; people would be unable to "escape" from their online past. With the increasing number of people that make use of the Web to share and consult information [7, p. 382] such concerns seem justified. In only a relatively short time, the quality and quantity of digital data storage and online accessible information have grown

\footnotetext{
${ }^{1}$ The Web is an application of the Internet. The content of this paper may also be applicable to other applications of the Internet, but to ensure clarity in this paper I have restricted my focus to the Web.
} 
explosively [13]. The dramatic increase and ease with which digital data can be stored have led from a situation that could be described as "forgetting-by-selection" due to limited storage space, to a "remembering-by-default" in our information systems. The technology of the Web is thus influencing what we remember and how we remember.

According to Dodge and Kitchin, stronger growing digital memory technologies mean that "memory should always be complemented by forgetting" [9, p. 441]. In the proposal for the European Union General Data Protection Regulation (GDPR) a "Right to Be Forgotten or Erasure" (R2BFE), article 17 (art. 17 GDPR) was developed in order to provide for such a counterbalance to digital memory. Ideally, the R2BFE aims to help individuals escape their past by targeting information that already exists in the public domain and that "with the passing of time becomes decontextualized, distorted, outdated, no longer truthful (but not necessarily false)" [3, p. 127].

Based on its title, the R2BFE seems to promise people a reinforcement of the forgetting of memories that concern them. However, "forgetting" is a concept that generally takes shape in the human ${ }^{2}$ memory process of a singular agent ${ }^{3}$ while the R2BFE is directed at information that is shared among agents. The information available on the Web is not only of interest to the agent that is the object of the information, but also to the people that share and consult that information. This makes the concept of human forgetting in such a context challenging to implement.

Taking into account the importance that is assigned to forgetting within the R2BFE, it is important to evaluate how successful the R2BFE could actually be in its implementation of forgetting. By analyzing the R2BFE in the light of human memory processes and the role that the Web plays in human memory, we can get a better idea of the possibilities of how to implement such a concept as human forgetting in relation to data on the Web and subsequently - and more importantly - where the R2BFE may lose out or may be at risk of affecting more than is necessary other interests that are at stake.

So let us assume that art. 17 GDPR was written with the intention to actually provide a "right to be forgotten", then my fundamental question is: to what extent can we consider the R2BFE to be a successful proposal for implementing forgetting on the Web? To answer this question I will first describe the concept of "memory", the use of external memory sources and the manner in which agents use the Web as a shared memory. This is followed by a section on "forgetting". Next I will analyze how the R2BFE implements forgetting in the online shared memory and conclude to what extent the implementation of "forgetting" by the R2BFE does justice to the concept of human forgetting and on which fronts it misses out.

\footnotetext{
2 The focus on human memory and forgetting does not mean that humans are the only species that forget or have a memory process. However, because "biological memory" seemed too broad a topic for this paper and the R2BFE is aimed at human agents, the concepts of memory and forgetting are framed here in human terms.

3 The agent can be any gender type, including gender $\mathrm{X}$ (the third legal gender, for example, in Australia). Because truly gender neutral pronouns like "xe" are still regarded as uncommon, I will refer to an agent as "he/him/his", although this use of the masculine gender is meant to include all options.
} 


\section{Memory, External Memory and Transactive Memory}

If we want a Web that forgets, we need to get an idea of the semantic content of "forgetting", and for that, we need to know what memory entails. The manner in which we use "our" memory systems has changed and is still changing over time due to technological developments i.e. on the level of information management. Additionally, our memory depends not only on what a single agent can do, but also on what agents can do through social interaction.

To highlight the functioning of our memory in relation to the Web, this section consists of three parts: the concept of "memory", the manner in which we externalise our memory by the use of tools and the manner in which we use the Web in a socially interactive way as a form of external memory, the transactive memory.

Firstly, the concept of "memory". "Memory" is a complex concept that has given rise to much thought in various academic fields [17]. The term "memory" is commonly used for both biological (human) entities and computers (Cf. [6]). This does not mean that these types of memory systems are the same: on the contrary, they differ a lot. For example, the electronic memory system seems far more apt for storing information than the human system; if they would be the same, there would possibly not even be a desire for the R2BFE. However despite (big) differences in its application, the term "memory" is considered applicable for diverse systems because they have something in common that is inherent to memory systems:

"Any memory system - whether physical, electronic, or human - requires three things, the capacity to encode, or enter information into the system, the capacity to store it, and - subsequently - the capacity to retrieve it." [2, p. 5]

These three elements interact: the manner of encoding determines what and how something is stored, which will in turn determines what can be retrieved [2, p. 5]. The concrete functioning of these three elements can differ according to the type of memory system and entity, but all necessarily comprise the same three elements.

With regard to the human memory process, the brain has special strategies to enhance its memory process with the help of environmental factors. This brings us to the second topic: the use of external tools in the human memory process. Clark points out that "[o]urs are (by nature) unusually plastic and opportunistic brains whose biological proper functioning has always involved the recruitment and exploitation of nonbiological props and scaffolds" [8, p. 86]. The use of external tools to increase our cognitive abilities has long been related with the human species: writing is one way that many of us use to think and reflect upon our own thoughts or to remember [8, p. 5]. By using tools to store, alter, combine and transform information we can deal with complex problems and large amounts of information in ways that would be impossible for or require a lot of time and energy from our biological "naked" brains [8, p. 78]. The human brain is good at learning how to use environmental factors and instruments optimally so as to minimize weaknesses like its generally limited memory capacity [8, p. 74-75]. External memory sources can complement this limited capacity by diminishing the amount of information that the biological memory needs to process and store [8, p. 67]. 
One of these potential external memory sources is the Web. The Web as external memory source differs fundamentally from many other information resources, due its "ontological features" [12, p. 186]. Information on the Web is not bound to a specific physical information source. Digital information is aspatial [19, p. 412]. Because of this, it is easily transported [5, p. 1112]. It is also generally a nonrivalrous good: meaning that the consumption of a good by one person, does not diminish the possibility of its use by others [19, p. 411]. The ontological features of the Web determine the effort that we need to undertake to get access to the information that it contains [12, p. 186]. Together these features determine the degree of "ontological friction" [12, p. 186] of the Web as "infosphere": "the forces that oppose the information flow within (...) the infosphere, and hence (...) the amount of work required for a[n] (...) agent to obtain information" [12, p. 186]. The Web's ontological frictions are low compared to offline, and especially analogue, information sources. Only a device to access the Web is needed and then one can access it almost from anywhere at any time while, if we want for instance to access information in an analogue library, we needs to physically access the building.

Besides its easy accessibility, the digital information on the Web is also easy to store. The storage capacity of digital devices has grown exponentially over the past decade and the quality loss of the stored information is relatively minor [13, p. 67]. Moreover, digital information can be multiplied, and copied easily and flawlessly, without loss of quality and is therefore infinitely expansible [19, p. 411] which can lead to a certain persistence [5, p. 1112]. Additionally the Web provides for easy retrieval of information. By means of search engines and other functions we can scan the Web for certain topics in a great level of detail, and generally we are able to find the desired information even when using vague keywords and search terms [8, p. 153].

The Web is not just a useful tool for us with regard to our memory processes as a singular human being. This fact brings us to a third topic: the Web as a socially interactive memory tool. The Web has a peculiar informational character compared to "classic" resources like libraries and archives. It has a decentralized and anarchic nature [8, p. 149-150]; it is an information source that is created by the minds of many. Everyone with an appropriate device can add information to the Web and often websites are publicly accessible. This gives the Web a special status; it can function as a "transactive memory system" [16].

Wegner ${ }^{4}$ introduced the term "transactive memory" to describe the processing and structuring of information within a group of people [20, p. 185]. Transactive memory is "a set of individual memory systems in combination with the communication that takes place among individuals" [20, p. 186]. The notion of transactive memory is based on an analogy between informational group processes and the individual memory process [20, p. 185]. In this sense, the processes of encoding, storage and retrieval have "both internal and external manifestations" [20, p. 188]. People can encode and store information both in themselves and in external sources (including other agents)

\footnotetext{
${ }^{4}$ Daniel M. Wegner, the founding father of the notion of transactive memory, sadly died in July 2013 while I was writing this paper.
} 
and they can retrieve the information they need from these sources and - if made available - from other agents and their external sources [20, p. 188]. Access to a transactive memory allows agents to enhance their own (external) memory stores and to retrieve information that they themselves have never encoded and stored [20, p. 188]. The Web is used as such a transactive memory. Sparrow, Liu and Wegner, in recent research stated that the Internet - this includes the Web - "has become a primary form of external or transactive memory, where information is stored collectively outside ourselves" [16, p. 776]. The features of the Web enable people to easily reach and consult online memory stores, especially search engines and the like effectively facilitate the retrieval of online information [16, p. 776]. The advantage of search engines is that the user does not need to know the location of the information, but that knowledge of the general label is often sufficient. Sparrow at al. conclude that "[w]e are becoming symbiotic with our computer tools, growing into interconnected systems that remember less by knowing information than by knowing where the information can be found" [16, p. 778].

Reciprocal use by agents of each other's memory leads to a transactive memory system "that is larger and more complex than either of the individual's own memory systems" [20, p. 189]. Information from different sources can be linked together and lead to new insights [20, p. 197]. Transactive memory systems thus influence what a group of people remembers and what they individually believe to be true [20, p. 191]. Consequently the Web as transactive memory can influence how we remember and what we remember.

The Web is thus to be seen as a transactive memory system that has a lower degree of ontological friction than traditional libraries or archives. This difference is significant and may lie at the base of a desire to have a R2BFE. The Web's large storage capabilities, its easy access and the fact that everyone can add information to the Web make it into a massive external transactive memory source that memorizes by default. The chief question therefore is: how can the Web "forget"?

\section{$3 \quad$ Forgetting}

The R2BFE claims to be - as part of the title of this paper states - a right "to be forgotten". But what is "forgetting"?

Generally forgetting is described in relation to human agents. Human agents can be considered "to forget" in both a literal and metaphorical manner. ${ }^{5}$ For the purpose of this paper I will only focus on the literal form of forgetting where forgetting is the

\footnotetext{
${ }^{5}$ Making a strict distinction between these two elements of forgetting is not always possible, because they can relate to each other in a reciprocal manner. Metaphorical forgetting relates to instances that are generally described as "forgive and forget": certain information is not literally forgotten by the agent, but the agent will not actively use the information for current decisions. An agent makes a conscious choice to neglect specific memories in certain cases. However, if by this behaviour the information is used less and less and eventually loses its meaningfulness for the agent, there is a fair chance that the information will also literally be forgotten.
} 
occurrence of a glitch ${ }^{6}$ in the memory process, a "fail[ure] to remember" [6]. Forgetting can occur as a result of failures in any of the three elements of the memory process. It can be the loss of acquired information (storage), the inability to retrieve stored information (retrieval) and the deterioration of correspondence between the acquired and the retrieved information (encoding) [10, p. 100]. Many nuances are possible, like only losing part of the stored information, being able to retrieve information only partially, a temporary inability to receive certain information or a combination of different failures [10, p. 101]. For the purpose of this paper, I will focus only on the three memory process elements (storage, retrieval and encoding) - although I duly note that reality is far more complex and nuanced than such a basic model.

As stated, forgetting is a glitch somewhere in the three process elements of memory. However not every piece of information is remembered and forgotten equally. The likelihood of a glitch in the encoding, storage and/or retrieval of a specific piece of information in the memory depends on three main factors: the passing of time, the meaning of the information and the regularity with which the information is used [10, p. 100/101]. On the one hand, meaningful and repeatedly used information generally has a longer endurance in the memory; on the other hand, the memory of a certain piece of information generally gets weaker over time [10, p. 100-101] $]^{7}$ These factors can strengthen or weaken each other. For example, meaningful information may be used more often and thereby remembered better. Additionally, information often loses its value for us over time [1, p. 390], which leads to the increasing chance that it is forgotten eventually. The factors "meaning", "use" and "time" together influence if and till what extent an agent will remember or forget a specific piece of information.

What forgetting entails in relation to human agents is relatively clear. But as described in the previous section of this paper, human agents can extend and share their external memory with others. With regard to the use of external personal memory and external transactive memory, the notion of forgetting becomes increasingly difficult.

Let us start with forgetting in relation to the use of an external memory source by a single human agent. When using an external memory source, the storing of information requires an action of the agent to actively select and encode the information that he wants to store. This extended-by-externalization memory process can show signs of glitches in the extended memory process when the agent interacts with his external memory source: for example, if the agent is unable to retrieve information from an external memory source (i.e. he loses a paper notebook) or if the information in it becomes intelligible (i.e. a cup of coffee is spilt over some of the notebook's pages). Such glitches can be viewed as metaphorical forgetting in the extended memory process. If an agent wants to "forget" information in his external memory store, he will need to actively effectuate the "forgetting" in some way. He could do this by, for instance, destroying the externally stored information or by making sure that it is irretrievable. With the use of most external memory stores (like notebooks), the storing faculty changes from a human memory store that automatically forgets by default into

\footnotetext{
6 "Forgetting" may also be seen as a feature of the memory process that generates storage space.

7 Again, this is a basic model of a more complex and nuanced reality.
} 
an external memory store that automatically remembers by default. The "digital turn" of information even strengthened the remembering by default in external memory stores. The previously needed forgetting-by-selection processes due to limited storage space are becoming increasingly unnecessary due to technological developments that enable the increased storage space of digital information [18, p. 349].

Understanding "forgetting" in relation to an external transactive memory is even more challenging, because it does not concern a single agent but multiple agents that may all have different interests at stake. When the transactive memory is stored externally, and especially when in digital format, the transactive memory system will automatically remember by default. The social base of the transactive memory makes it increasingly difficult to see how "forgetting" could work in such a system. The system depends on both the input and output of all agents and is often not under the control of a single agent. All agents that consult the external transactive memory (and not only the ones that add information to it) and/or that are the object of these "memories" have an interest in what is encoded into the memory and what is not, or what is "forgotten". This means that the factors of "meaning", "time" and "use" in the transactive memory differ in their relative importance with regard to the "forgetting" of a specific piece of information depending on the agent in question.

The challenge for the R2BFE is thus to implement the concept of singular human forgetting into the external transactive memory that forms a memory source for multiple agents.

\section{$4 \quad$ R2BFE and Online Transactive Memory}

The term "forgetting" in the R2BFE implies human memory, but clearly the R2BFE is not aimed at human memory as such or else it would imply that there is a right to meddle in other people's brains. Rather, the R2BFE is part of the GDPR-proposal and therefore has to be read in the context of personal data protection and information technology systems. The R2BFE, art. 17 GDPR, gives people the "right to obtain from the controller the erasure of personal data relating to them and the abstention from further dissemination of such data" (art. 17 (1) GDPR). The R2BFE thus provides individuals with a right to interfere with digital information that is controlled by others, called data controllers (art. 4 (5) GDPR), and is located on an external memory source. Simply put, the data controller's external memory sources need to "forget".

As pointed out in section two of this paper, external memory sources play an important role in human memory processes because our brains use them as external tools to enhance our memory. Most external memory sources (like stored emails) will have a personal instead of a public nature. Such personal external memory stores cannot be targeted by the R2BFE; art. 2 (2)(d) GDPR states that the processing of personal data "by a natural person without any gainful interest in the course of its own exclusively personal or household activity" falls outside the scope of the GDPR. This is the "household exemption" [4, p. 5]. The target of the R2BFE is thus the external memory shared among people (transactive memory) that lies outside the household. A part of the transactive memory systems is thus safeguarded by the household exemption if it 
can be considered part of the "household" activity. The scope of what is intended by "household" is therefore very important. The current idea of what is to be considered "household" use includes for a major part the data made accessible by individuals to friends on social networking sites (SNS). The Article 29 Working Party states that many SNS users "operate within a purely personal sphere, contacting people as part of the management of their personal, family or household affairs" [4, p. 3]. However, some exceptions are mentioned: the processing of data on SNS is not considered to be household use when activities extend beyond purely personal or household activity (for instance when the user acts on behalf of a company), when the access to the profile information extends beyond self-selected contacts or when the information is subject to liability under general provisions of national law (such as defamation) [4, p. 5/6]. Due to the household exemption, a large part of the data on social networking sites fall outside the scope of the R2BFE.

With regard to personal websites, the European Court of Justice (ECJ) decided in the Lindqvist ruling that activities on a personal website are not covered by the household exemption when the information on a website could be viewed by an indefinite number of people $[11, \S 47]$. Here we enter the realm of the online transactive memory.

Additionally, the R2BFE tries to account for some of the ontological features of online data - the fact that they can be easily copied, multiplied and transported - in art. 17 (2) GDPR. Art. 17 (2) GDPR deals with third parties who have copied and reproduced data that is challenged by the R2BFE: it compels data controllers to inform third parties that are processing data that were originally published under the responsibility of the data controller, that the subject of the data wants the data to be erased. When the data controller has authorised publication by third parties, the data controller is considered responsible for those publications (art. 17 (2) GDPR).

The household exemption and the Lindquist ruling show that the group of people that can get access to the data play a important role for the criteria whether a certain information source - an external memory source - falls within the scope of the GDPR. The main types of memory that can be targeted by the R2BFE are thus publicly accessible and/or business oriented external transactive memory systems; transactive memory systems that have a big reach and hence can have a substantial group of people that have a legitimate interest in the content of that transactive memory.

The challenge for the R2BFE is to implement "forgetting", a concept that relates to the singular human memory, into a shared transactive memory system that transcends the memory of a single human being. The interests at stake with regard to a specific piece of information expand from one agent to a group of agents with diverse interests. The human memory metaphor can provide guidance on how to implement "forgetting" in online transactive memory systems. It can give guidance on which aspects should play a role in the balancing of interests of the different agents that are stakeholders with regard to the content of an online transactive memory system. In order to evaluate to what extent the R2BFE relates to the concept of human forgetting and implements this notion, I shall review the manner in which the relevant paragraphs of the R2BFE deal with this particular challenge. 
The R2BFE targets personal data that relate to a certain individual. Art. 17 (1) GDPR states the grounds on which people have a R2BFE in sub-paragraphs (a) through (d): sub-paragraph (a) grants agents a R2BFE when it concerns data that "are no longer necessary in relation to the purposes for which they were collected or otherwise processed", (b) determines that agents can invoke their R2BFE when the processing of data is based on consent and the agent withdraws this consent or when the period for which the consent is given is expired, (c) entitles agents to a R2BFE if the agent objects to the processing of personal data in accordance with art. 19 GDPR. The data has to be erased, unless the data controller can show compelling legitimate grounds that override the agent's interests (art. 19(1) GDPR), and (d) requires the erasure of data when the processing of the data is not conform the GDPR.

These subparagraphs show the balance of interests between an agent that wants information "forgotten" and the data controller that wants to retain the information. In determining this balance of interests the elements of "meaning" and "time" play a role; the data need to have meaning in order for the data controller to keep it in his external memory source. Art. 17 (1)(a) GDPR contains a temporal and meaning reference that suggests that, with the passing of time, information may lose its relevance for a data controller, in which case the individual's R2BFE prevails. This subparagraph restricts a processor's data collection to data that serves the specified purpose for data processing.

The exceptions to what may be forgotten by the enforcement of the R2BFE are also important. These are listed in art. 17 (3) GDPR. Data can be retained (a) when the data is necessary for exercising the right of freedom of expression, (b) for reasons of public interest in the area of public health, (c) historical, statistical and scientific research purposes, and (d) for compliance with legal obligation to retain data and in specific cases described in art. 17(4) GDPR. As these exceptions show, if information has public value the data can be retained. What is important to underline here, is that these exceptions acknowledge the meaning of the data for third parties that consult or make use of the external transactive memory and thus acknowledge their interest with regard to a certain set of topics (the data need to be important for the freedom of expression, public health, historical and/or scientific value).

Additionally the formulation of art. 17 (4)(a) GDPR is interesting: "for exercising the right of freedom of expression". The use of the verb and its conjugation "exercising" suggests that data can be retained if they are part of an ongoing activity of freedom of expression. The question then is: when does this action end? The temporal scope of a freedom of speech activity is an interesting issue, and it is worthwhile debating if the R2BFE is implemented and retains this formulation. Such a discussion however exceeds the scope of this paper.

The R2BFE has some elements that correspond with important factors in the human forgetting process. The most prominent one is the meaning of the information: it is included in terms of whether the data are necessary (meaningful) for the data controller, if the data should be retained due to its public importance (art. 17 (3) GDPR; freedom of expression, public interest in the area of public health, historical, statistical and scientific research purposes) and the meaning of the information for the individual that wants the data to be forgotten. The time-factor of forgetting is less prominent, 
it is only a factor in art. 17 (1)(a) GDPR. It is notable that any element that acknowledges the importance of the "use" of data in relation to forgetting, the frequency with which the data are used, is missing altogether.

The "forgetting" that is implemented by the R2BFE is initiated by the individual who wants certain data to be forgotten. The R2BFE is thus an externally initiated and enforced form of "forgetting". As the name of the article already indicates, it is a right to be forgotten and to erasure. The first paragraph shows that "erasure" is the dominant feature of the article, because the R2BFE gives individuals the "right to obtain from the controller the erasure of personal data". By erasing information the R2BFE operates on the storage level of the transactive memory process and thereby applies a black-and-white notion of forgetting: data are either erased or not. This is in contrast with the concept of human forgetting which can also play a role at the process levels of encoding and retrieval, and may vary in gradations of forgetting: in human terms, information can be partially or temporally forgotten.

However, there is (minor) potential in the in the second and fourth paragraph of the article of the R2BFE for it to be applied to the two levels of retrieval and/or encoding. Art. 17 (2) GDPR compels data controllers "to inform third parties which are processing such data, that an individual requests them to erase any links to (...) that personal data". An erasure of hyperlinks would be an interference with the transactive memory process on the level of retrieval. The question, however, is whether a controller can request third parties to remove the links while retaining the information on his own website due to one of the exceptions of art. 17 (3) GDPR. In this situation, the information would be more difficult to retrieve, but not impossible to retrieve for people who know what they are looking for and where to look. Also, it is unclear whether an individual could use the R2BFE only to have links removed, and not the content itself: for instance, if the agent wants to retain the content for sentimental reasons, but not draw attention to it because it is outdated. ${ }^{8}$

Art. 17 (4) GDPR mentions several cases where data controllers can refrain from erasing data, but have to restrict its processing. Sub-paragraph (b) and (c) are interesting in the light of "forgetting". A controller is allowed to restrict the processing of data instead of erasing them, when (b) the data controller needs to maintain the data for the purposes of proof, (c) the processing of the data is unlawful, but the individual requests the restriction of the use of the data instead of erasure. In the case of (b) the interference of the R2BFE works on the levels of retrieval and encoding in the transactive memory process: the data may only be retrieved for the purpose of providing proof and no additional information may be encoded, nor may the already encoded information be altered. Roughly the same goes for (c), with the difference that the request for the retention of the data is initiated by the individual. However, paragraph

\footnotetext{
${ }^{8}$ What plays an important role here is whether a search engine can be considered to be a data controller. This is one of the central legal questions in the Google versus Spain case (case number C-131/12) that is currently still under discussion by the ECJ. Advocate General Jääskinen stated in his advice to the court that search engines cannot be considered data controllers. The question is: will the court hold the same interpretation of the law? Opinion of Advocate General Jääskinen, 25 June 2013, Case C-131/12.
} 
four of the article seems to be focused on internal company processes and not aimed at online publicly accessible content.

In sum, the R2BFE is largely concentrated on erasure on the storage level and only minimally allows for other - more fine-grained - applications of "forgetting" that may deal with the same issue at hand. Possibilities to use implementations of "forgetting" on the process levels of encoding and retrieval are the exception rather than the rule.

\section{$5 \quad$ Learning from Forgetting by the Human Memory}

As stated earlier, the human memory metaphor can provide valuable guidance on how to implement "forgetting" in online transactive memory systems. Its rich mechanisms of forgetting provide for a better understanding of which aspects can and should play a role in the balancing of the interests of different stakeholders in online transactive memory systems and give guidance for implementations of more gradual and balanced forms of "forgetting". A straightforward erasure of data is a too narrow understanding of "forgetting".

The "forgetting" implemented by the R2BFE needs to take place somewhere in the transactive memory process of encoding, storage and retrieval. The element of retrieval seems to be a very significant part in the use of the Web as transactive memory; people can get access to information they did not even know existed through the use of search engines [16]. Cues in search engines can lead to search results that individuals were not originally looking for and search results can provide for new insights by showing information that can be combined serendipitously. The R2BFE would obtain means to more nuanced implementations of "forgetting" if it were to be able to impede for instance, the retrieval of information by search engines. Links could be erased or moved to a harder-to-access area (e.g., an area that requires a user login or an area that can be found only with the use of very specified retrieval cues in a search engine), or certain results in search engines could be downranked. ${ }^{9}$

On the level of encoding, there may be possibilities to anonymize, pseudonymize or encrypt information or certain - identifying - parts of the data (this in return will influence the extent of retrieval of the information). Additionally, one can think of encoding more contextual and actual information with memory items, so that people who consult the transactive memory view updated instead of possibly outdated information. Such forms of forgetting or updating of the memory could be a less heavy and more goal-effective means of forgetting than erasure of data on the storage level.

Hold-ups in the retrieval process or obscuration in the encoding process would obviously interfere with the interests of individuals who try to consult the transactive memory. However, they will have a greater chance of retrieving the "forgotten" information or at least part of it - with some effort - than would be the case if the information were to have been erased altogether. An implementation of forgetting on the level of encoding and/or retrieval would be therefore a less extensive integration

\footnotetext{
${ }^{9}$ The search engine Google uses ranking algorithms to deal with certain issues, see for example http://googleblog.blogspot.nl/2010/12/being-bad-to-your-customers-is-bad-for.html (last accessed 8 November 2013).
} 
of forgetting in the online transactive memory than the erasure of the content itself. Using less far-reaching measures than the plain erasure of data could be beneficial for the interests of others. It could include access to: the historical and scientific value of information, the freedom of speech, or personal interests of others. Also an individual self can have a need for different "grades" of forgetting rather than plain erasure.

With an eye on the balancing of interests between the individual who wishes certain personal information to be forgotten and the users of the transactive memory, it will be worthwhile to pay attention to factors that influence the memory process - of which "meaning", "time" and "use" are the most important ones.

In its current form, the R2BFE neglects the frequency with which information is used as a factor that affects the forgetting process. Allocating a role to the "use" of information as a factor that either enforces or prevents "forgetting" can be a helpful element in weighing up the balance of various interests. "Forgetting" information on a website that is hardly viewed makes more sense than "forgetting" information that is consulted several hundreds of times a day. Integrating "use" as a factor could benefit the interests of third parties, which as consulting parties are now only taken into account by means of the exceptions to the R2BFE. "Use" as a factor that could oppose "forgetting" provides a possibility for concrete evaluation: measuring how often something is viewed is technically easy on the Web. Additionally giving more attention to the factors "time" and "meaning" - not just for the data controller, but also for the third parties who consult the transactive memory - could help in providing the R2BFE with the right tools to oversee a good balance of interests.

Additional and especially technical research is needed to obtain better understanding of the possibilities of how to implement more sophisticated forms of "forgetting" in the online transactive memory. This will enable us to shape and implement a R2BFE that is more fine-tuned and based on a more nuanced process of forgetting that will be better equipped to balance between an individual's need to be forgotten and the other interests at stake.

\section{Conclusion}

Technology has changed and is still changing our internal and external memory processes. The Web - as discussed in this paper - is one of those technological developments that affects the way we remember and what we remember in fundamental ways. We have moved from external memory stores with limited storage space that therefore had to "forget"-by-selection to memory stores with ever-growing storage space that "memorize"-by-default.

The R2BFE aims to deal with the possible negative consequences of the shared online memory by providing people with a means to have some of their data in the online transactive memory "forgotten". Implementing "forgetting" into such systems is difficult, because the concept of forgetting originates from a single agent's internal memory process. Instead of functioning as forgetting within a singular agent, the R2BFE has to implement forgetting in an external transactive memory that affects the interests of multiple agents. The current wording of the R2BFE does this with limited success. 
The manner in which the R2BFE implements "forgetting" is mainly a "right to erasure" of content and operates thereby mainly on the storage level of the external transactive memory process. The present possibilities for the R2BFE to operate on the level of encoding and retrieval are very limited. The R2BFE thus uses a narrow interpretation of "forgetting". While "erasure" is black-and-white (information is either erased or it is not), "forgetting" provides for a big greyscale. Additionally, of the factors that influence the occurrence of forgetting, only the "meaning" of the information for diverse agents plays a significant role. "Time" as a relevant factor to either enforce or avert forgetting is only marginally acknowledged in the R2BFE and "use" is not acknowledged at all.

The R2BFE could be transformed into a more successful implementation of "forgetting" in the external transactive memory if it were to draw more heavily on the mechanisms of human forgetting. Despite the fact that the human memory is based on a single agent, the human concept of memory shows us that there are more levels in information processes in which we could interfere to achieve "forgetting". Instead of the plain erasure of information we could interfere on the level of encoding or retrieval, which can also lead to successful "forgetting".

Furthermore, the factors "meaning", "time", and "use" provide for a better understanding of which aspects play a role in every agent's relation to (memorized) information and thereby to information in the external transactive memory. These factors give guidance as to which aspects can and should play a role in the balancing of the interests of the different stakeholders in online transactive memory systems: not just the agents that are the object of the memory or the data controller, but also, very important, the third parties that use the external transactive memory as memory and may rely on it.

Acknowledgments. This research is conducted within the Privacy and Identity Lab (PI.lab) and funded by SIDN.nl (http://www.sidn.nl). Additionally I would like to acknowledge the help of the IFIP Summer School participants, my anonymous reviewers, and Bert-Jaap Koops, Ronald Leenes and Diane Whitehouse by providing me with very helpful and much appreciated comments and suggestions.

\section{References}

1. Ambrose, M.L.: It's About Time: Privacy, Information Lifecycles, and the Right to Be Forgotten. Stanford Technology Law Review 16, 369-422 (2012)

2. Anderson, M., Eysenck, M.W., Baddeley, A.: Memory. Psychology Press, London (2009)

3. de Andrade, N.N.G.: Oblivion, The Right to Be Different from Oneself. Reproposing the Right to Be Forgotten. In: VII International Conference on Internet, Law \& Politics. Net Neutrality and Other Challenges for the Future of the Internet, IDP. Revista de Internet, Derecho y Política, vol. 13, pp. 122-137 (2012)

4. Article 29 Working Party, WP 163, Opinion 5/2009 on online social networking

5. van den Berg, B., Leenes, R.: Audience Segregation in Social Network Sites. In: Proceedings for SocialCom2010/PASSAT2010 (Second IEEE International Conference on Social Computing/Second IEEE International Conference on Privacy, Security, Risk and Trust), pp. 1111-1117. IEEE, Minneapolis (2010) 
6. Concise Oxford English Dictionary, 11th edn.

7. Castells, M.: The information Age: Economy, Society, and Culture, 2nd edn. The Rise of the Network Society, vol. I. Wiley-Blackwell, Chichester (2010)

8. Clark, A.: Natural-Born Cyborgs: Minds, Technologies, and the Future of Human Intelligence. Oxford University Press, Oxford (2003)

9. Dodge, M., Kitchin, R.: 'Outlines of a World Coming into Existence': Pervasive Computing and the Ethics of Forgetting. In: 34 Environment and Planning B: Planning and Design, pp. 431-445 (2007)

10. DuDai, Y.: Memory from A to Z. Oxford University Press, Oxford (2004)

11. European Court of Justice, case C-101/01, Criminal proceedings against Bodil Lindqvist

12. Floridi, L.: The Ontological Interpretation of Informational Privacy. Ethics and Information Technology 7(4), 185-200 (2005)

13. Mayer-Schönberger, V.: Delete: The Virtue of Forgetting in the Digital Age. Princeton University Press, Princeton (2009) (used print: 2011)

14. Proposal for a General Data Protection Regulation, COM(2012) 11 final (January 25, 2012), Download: http: / / ec .europa.eu/justice/data-protection/

document/review2 012 /com_2012_11_en.pdf (last accessed November 8, 2013)

15. Rosen, J.: The web means the end of forgetting. The New York Times 21 (2010)

16. Sparrow, B., Liu, J., Wegner, D.M.: Google effects on memory: Cognitive consequences of having information at our fingertips. Science 333(6043), 776-778 (2011)

17. Sutton, J.: Memory. In: Zalta, E.N. (ed.) The Stanford Encyclopedia of Philosophy, 2012th edn. (Winter), http: / plato.stanford. edu/archives /win2012/

entries/memory/ (last accessed November 8, 2013)

18. Szekely, I.: The Right to Forget, the right to be Forgotten; Personal Reflections on the Fate of Personal Data in the Information Society. In: Gutwirth, S., Leenes, R., De Hert, P., Poullet, Y. (eds.) European Data Protection: In Good Health?, pp. 347-363. Springer Netherlands (2012)

19. Vafopoulos, M.: Being, space, and time on the Web. Metaphilosophy 43(4), 405-425 (2012)

20. Wegner, D.M.: Transactive memory: A contemporary analysis of the group mind. In: Mullen, B., Goethals, G.R. (eds.) Theories of Group Behavior, pp. 185-208. Springer, New York (1986) 